\title{
FMEA and Alternatives v/s Enhanced Risk Assessment Mechanism
}

\author{
Gunjan Joshi \\ Senior Engineer, Honeywell \\ Aerospace, \\ Hyderabad, India
}

Himanshu Joshi

JNTUH-CEH,

Hyderabad, India

\begin{abstract}
This paper starts with description of the widely used risk assessment tool Failure Modes \& Effects Analysis. The paper then later examines some of the alternative methods for risk assessment and their advantages. In this work, we also point out the advantages of using Six Sigma in Risk Assessment and propose a novel technique which would overcome the restrictions of existing Risk Management tools.
\end{abstract}

\section{General Terms}

Failure Modes \& Effects Analysis, Risk Assessment.

\section{Keywords}

FMEA, Risk Assessment, Risk Management, FMEA Alternatives, Cause \& Effect, Tree Analysis, Six Sigma

\section{INTRODUCTION}

FMEA has been most widely used tool irrespective of occupation and status to determine weakness in a proposed new layout/process/product or to improve an existing layout/process/product.

FMEA was first used in 1960s for the Apollo Missions by NASA to record and assess design related risks [1]. There have been many other adoptions of FMEA since then. Some of them are listed below.

- Design Review by Failure Mode (DRBFM) developed by Toyota which has all main features but skips out scoring system [2]

- Failure Mode, Effects and Criticality Analysis

(FMECA) used by Nuclear, Aerospace and Automotive Industry which uses real probabilities for numeric ranking [3]

As one of the erstwhile systemic proactive method for failure analysis, the FMEA tool is universally a part of Analysis and Implementation phases of a project. Despite its far-flung use the tool has its own shortcomings.

The rest of this paper is organized as follows.

Section 2 provides an introduction to FMEA, FMEA advantages, FMEA linkage to other process tools and FMEA limitations.

Section 3 presents FMEA alternatives.

Section 4 presents a summary of improvements that can be done in Risk Assessment with the help of Six Sigma techniques.

Section 5 presents a solution for FMEA restrictions and Section 6 concludes the work and highlighting some perspectives as future works.

\section{FMEA - INTRODUCTION \& ANALYSIS}

\subsection{Definition, Purpose \& Types}

FMEA is a structured approach to:

- evaluate a process/product to identify where $\&$ how it might fail

- estimate the risks of specific causes associated with these failures

- assess impact of these failures

- minimize the impact and chance of these failures by taking the appropriate actions

- identify parts/products in systems that majorly call for a change

\section{FMEA purposes:}

- discover potential failure modes and the severity of their consequences

- prioritize the potential deficiencies

- develop action plan to focus on reducing/preventing risks (FMEA forms the basis for Control Plans)

- assess impact of proposed change in the design/system

- identify areas that are most important to customers

Types of FMEA:

- Process: Used to analyze manufacturing, assembly \& administrative processes

- System: Used to analyze system and sub-systems during early concept $\&$ design phase

- Design: Used to analyze product designs prior to production

\subsection{Advantages}

The FMEA is used to capture potential failures/risks \& impacts and prioritize them on a numeric scale called Risk Priority Number (RPN) which ranges from 1 to 1000 . RPN is obtained by multiplying Severity, Occurrence \& Detection. Each of Severity, Occurrence \& Detection is identified on a scale of 1 to 10 . This is a very sensible and effective method if executed punctiliously.

Figure 1 presents a sample FMEA form which is used to enter failure modes, their causes and actions taken for risk mitigation.

Figure 2 presents a sample FMEA rating scale. The teams using the FMEA develop a scale of their own. 


\begin{tabular}{|l|l|}
\hline Process or Product Name: & \\
\hline Responsible: & \\
\hline
\end{tabular}

\begin{tabular}{|l|l|}
\hline Prepared by: & \\
\hline Date: & \\
\hline
\end{tabular}

\begin{tabular}{|c|c|c|c|c|c|c|c|c|c|c|c|c|c|c|}
\hline $\begin{array}{c}\text { Process Step } \\
\text { / Input }\end{array}$ & $\begin{array}{c}\text { Potential } \\
\text { Failure } \\
\text { Mode }\end{array}$ & $\begin{array}{c}\text { Potential } \\
\text { Failure } \\
\text { Effects }\end{array}$ & $\begin{array}{l}\text { S } \\
\text { e } \\
\text { v } \\
\text { e } \\
\text { r } \\
\text { i } \\
\text { t } \\
\text { y }\end{array}$ & $\begin{array}{c}\text { Potential } \\
\text { Causes }\end{array}$ & $\begin{array}{l}\text { O } \\
\text { c } \\
\text { c } \\
\text { u } \\
\text { r } \\
\text { a } \\
\text { n } \\
\text { c } \\
\text { e }\end{array}$ & $\begin{array}{l}\text { Current } \\
\text { Controls }\end{array}$ & $\begin{array}{l}\mathrm{D} \\
\mathrm{e} \\
\mathrm{t} \\
\mathrm{e} \\
\mathrm{c} \\
\mathrm{t} \\
\mathrm{i} \\
\mathrm{o} \\
\mathrm{n}\end{array}$ & $\begin{array}{l}R \\
P \\
N\end{array}$ & $\begin{array}{c}\text { Actions } \\
\text { Recommended }\end{array}$ & $\begin{array}{c}\text { Actions } \\
\text { Taken }\end{array}$ & $\begin{array}{l}\text { S } \\
\text { e } \\
\text { v } \\
\text { e } \\
\text { r } \\
\text { i }\end{array}$ & $\begin{array}{l}\text { O } \\
\text { c } \\
c \\
u \\
r \\
r \\
e \\
\text { n } \\
\text { e }\end{array}$ & $\begin{array}{l}D \\
e \\
t \\
e \\
c \\
t \\
i \\
o \\
n\end{array}$ & $\begin{array}{l}\mathbf{R} \\
\mathbf{P} \\
\mathbf{N}\end{array}$ \\
\hline & & & & & & & & & & & & & & \\
\hline & & & & & & & & & & & & & & \\
\hline & & & & & & & & & & & & & & \\
\hline & & & & & & & & & & & & & & \\
\hline & & & & & & & & & & & & & & \\
\hline
\end{tabular}

Figure 1: Sample FMEA Form

\begin{tabular}{|l|l|c|}
\hline Probability of Failure & Possible Failure Rates & Rank \\
\hline Extremely High: Failure almost inevitable & $>=1$ in 2 & 10 \\
Very High & 1 in 3 & 9 \\
Repeated Failures & 1 in 8 & 8 \\
High & 1 in 20 & 7 \\
Moderately High & 1 in 80 & 6 \\
Moderate & 1 in 400 & 5 \\
Relatively Low & 1 in 2,000 & 4 \\
Low & 1 in 15,000 & 3 \\
Remote & 1 in 150,000 & 2 \\
Nearly Impossible & $<=1$ in $1,500,000$ & 1 \\
\hline
\end{tabular}

Figure 2: Typical FMEA rating scale

The advantages of FMEA are but not limited to:

- uncomplicatedness \& convenience of tool

- help perform design/trade-off analysis

- reduce possibility of similar failures in future

- minimize the costs in a project

- reduce development time

- minimize late changes

- improve the product/process quality, reliability \& safety

- reduce risk of critical or catastrophic issues

- increase user satisfaction

- availability of risk records for future developments/changes

- focuses on prevention

\subsection{FMEA link to other Process Tools}

The inputs to FMEA tool come from the Process Map, SIPOC and C\&E Matrix. The FMEA then identifies the risks and control action plans which are documented in Control Plan tool. The tools viz. SIPOC, FMEA, C-Matrix, Control Plan \& Process Map are "living documents" - which means that when one changes, the other documents should also be updated accordingly. The relationships among these documents are depicted in Figure 3.

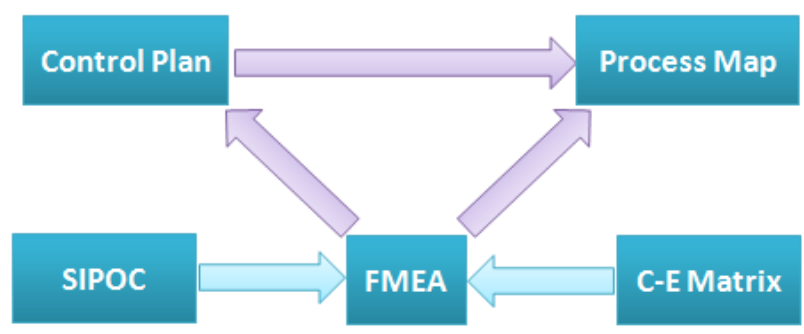

Figure 3: FMEA \& its linkage to other Process Tools

\subsection{Limitations}

As any other tool in the FMEA has its own pros and cons. Present below are some of the weaknesses of FMEA. 
- FMEA was first created for design related risks but now has different versions for process risks and system risks to overcome its original limitations. These versions have no linkage among them which allows overlooking of some failure modes

- FMEA is not an all-inclusive tool and misses certain risks. For example, in a healthcare context, FMEA has been found to have limited validity compared to another prospective hazard analysis method (Structured What If Technique, SWIFT) and retrospective approaches, with particular challenges around scoping and organizational boundaries

- FMEA often has repeated information

- The multiplication of Severity, Occurrence \& Detection numbers causes generation of false Risk Priority Numbers

- FMEA is only as good as the team since it requires brain storming and regular updating. Human errors should be examined

- Members of a team spend significant time debating about the rankings and collecting/entering details

- FMEA works best as a bottom-up tool and fails to identify all the failure modes if used for a top-down analysis

- FMEA does not provide any assessment or discover complex failures involving combination of failures

\section{FMEA ALTERNATIVES}

The alternative risk assessment techniques depend upon the industry in which they are used. Some of the other FMEA alternatives used are briefed below.

- Hazard and Operability assessment (HAZOP): It was created by ICI to analyze plant failures and effects. This is used in Chemical and Process. It uses Guide words instead of numeric content [4].

- Fault Tree Analysis (FTA): It was developed by Bell Laboratories in 1962 to evaluate the Minuteman I Intercontinental Ballistic Missile (ICBM) Launch Control System. It has been used since then as a powerful tool for to perform the probability calculations in nuclear and aerospace industries [5]. Many versions of FTA reliability software are available. FTA evaluates risk by tracing backwards in time or backwards through a cause chain. FTA also uses known failure mechanisms \& rates in risk determination.

It is complex but rigorous in capturing cascading failures or risks associated with simultaneous failures. Figure 4 presents a sample FTA template.

- Event Tree Analysis (ETA) is similar to fault tree but is more dynamic. It works by tracking forward in time or a casual chain to model risk. FTA is a deductive investigatory process where as ETA is inductive. ETA is widely used in Nuclear power plants [6]

- Cause Consequence Analysis: This technique combines the abilities of FTA \& ETA. It is useful for examining chain reactions of failure conditions [7].
There are also tools available to assess risks due to Human Factors since FMEA \& FTA cannot capture such failures.

Of the above mentioned techniques, some of them focus on preventions and some of them on remedies/mitigations. Some depend on past experience and some on published data which require specific tools, knowledge of statistics \& substantial computing energy. For example, Graphical methods are more structured and easy to visualize but requires complex software to navigate the tree.

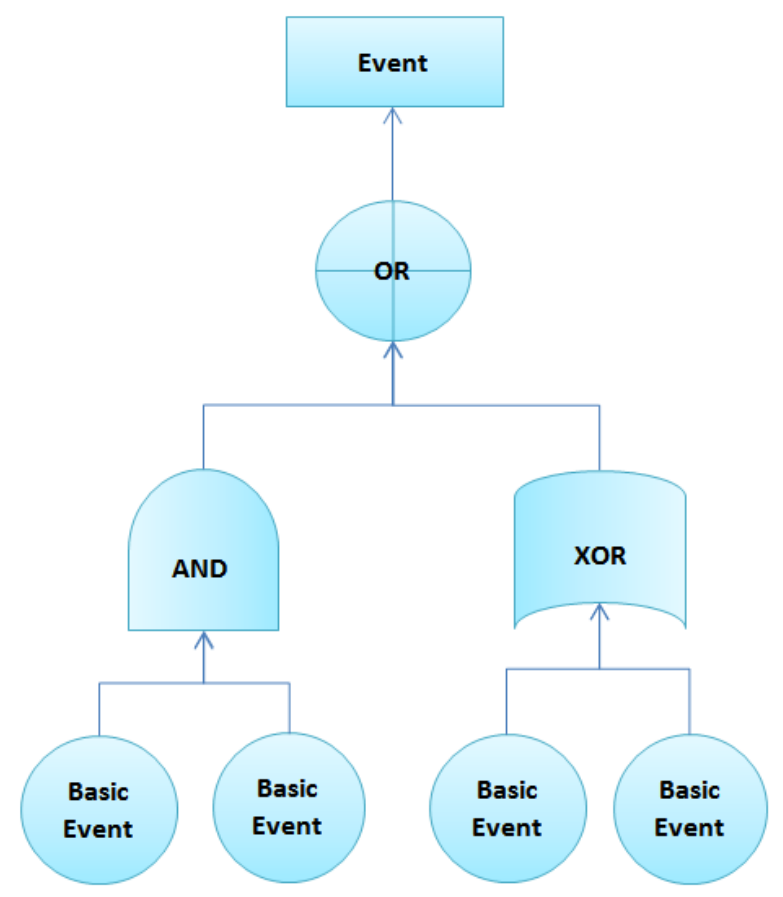

Figure 4: Sample FTA Template

\section{SIX SIGMA IN RISK ASSESSMENT}

Six Sigma can be used to optimize the risk assessment by using its structured methodology, i.e., DMAIC (Define, Measure, Analyze, Improve, Control). This approach not only fixes the broken process but also improves it. The process is described below.

a. Define:

In this phase, all risks should be identified correctly. One of the common mistakes that fail FMEA in define phase is to do an FMEA on an undocumented process.

FMEA works on a single why for each failure mode and does not dig deeper. A better method would be to use 5 Why's and generate a Cause-Effect tree structure.

b. Measure \& Analyze:

FMEA fails to identify the correct risk level because of the below reasons.

- restricted RPN scale

- incorrect assumptions

- other integrated systems not considered during evaluation In FTA, the "And" and "Or" gate relationships help analyze the risks more accurately.

c. Improve:

Linking action items to failures needs to be done clearly and prioritization of what steps to be taken next. One of the 
common mistakes is not effectively using a Control Plan. Also, linkage of Process FMEA \& Design FMEA would provide better picture of the risks and would provide Test Validation Plan \& Process Control Plan.

d. Control:

The outcome of FMEA should be a well defined set of actions to be taken for risk mitigation. Once the plan is made it needs to be monitored and updated as the time progresses. While carrying over FMEAs the risk levels should be corrected for the current state of the system.

\section{PROPOSED RISK ASSESSMENT MECHANISM}

The authors propose a new method of risk identification \& analysis which shall perform the following.

- use powerful statistical \& non-statistical tools to locate and eliminate root causes

- follow a 5 Why analysis instead of focusing on single point failure

- focus on defect removal

- reduce variability among the processes

Brainstorming needs to be done to find out all the possible causes of failures. Risks from similar past FMEAs can also be carry forwarded. Identify what could go wrong with each process step including Customer observed failures.

\begin{tabular}{|c|l|l|l|l|l|l|l|l|l|}
\hline S No. & Risk & \multicolumn{5}{|c|}{ Score by Individuals } & Total Score \\
\hline 1 & $<<$ Risk 1 > & & & & & & & & \\
\hline 2 & $<<$ Risk 2 > & & & & & & & & \\
\hline 3 & & & & & & & & & \\
\hline 4 & & & & & & & & & \\
\hline & & & & & & & & & \\
\hline
\end{tabular}

Figure 5: Risk Scoring Sheet

Once significant risks are obtained their causes need to be found. For each cause drilling down a little more by asking "5 Whys" and "What Ifs" should be done.

A Pareto analysis on the causes would provide us with the most important causes.

This method also shows that unimportant issues can turn out to be high priority ones. This method is easy to use and is a live document for product and process engineers.

FTA structures are complex but provide better analysis compared to FMEA. FMEA is simpler but misses out on certain risks/causes. Hence, the proposed method utilizes the best of both these worlds. The proposed method shall use simple linear structures to capture the data. An excel document or a spreadsheet would be a wonderful tool here.

Figure 6 presents the proposed tool/template for Risk Assessment. The document should be initially created during Concept phase and should be revised during design and testing phases. The document structure is flexible and allows capturing of causes without going into details. It can be easily re-used and modified. Color codes \& comments can be used

\begin{tabular}{|c|c|c|c|c|c|c|c|}
\hline S No. & Process Step & Failure Modes & Causes & Actions & Responsibility & Status & Comments \\
\hline \multirow[t]{6}{*}{1} & $<<$ Step $1>>$ & $<<$ Failure Mode 1 >> & $<<$ Cause $1>>$ & $<<$ Action $1>>$ & & & \\
\hline & & & & $<<$ Action $2>>$ & & & \\
\hline & & & & $<<$ Action $3>>$ & & & \\
\hline & & & $<<$ Cause $2>>$ & $<<$ Action $1>>$ & & & \\
\hline & & & & $<<$ Action $2>>$ & & & \\
\hline & & & & $<<$ Action $3>>$ & & & \\
\hline & & 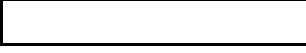 & & & & & \\
\hline
\end{tabular}

\section{Status Legend}

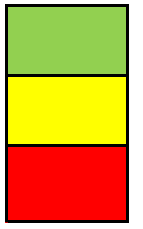

As per plan

Needs discussion, refer comments

Needs immediate attention/Re-baseline

For each risk identified, Customers and Stakeholders should be asked to provide scoring. The scores for each risk should be summed up and the most significant ones should go into Analysis. Below figure shows a sample scoring sheet.

\section{Figure 6: Proposed Risk Assessment Method}

for each section to trace the status of action items and highlight them.

With the above technique, Process and Design Risks are no more in separate instances of FMEA. Also, the C-E relationships from Customer \& Suppliers can be easily traced. The above method can also be used to generate an FMEA out of it. 


\section{RESULTS}

A simulation of Risk Assessment for "Improvement of Testing Efficiency" was done using the various Risk Assessment Techniques. Below are the features which were evaluated.

(1) Risk Prioritization

(2) DFMEA \& PFMEA linkage

(3) Coupling with Control Plan

(4) Involvement of External Risks

(5) Ease of Audit

(6) Relationship to C\&E

(7) Complexity \& Maintenance

(8) Reusability

For the various potential failures identified and assessed under each technique, the results can be found in tabular format in Figure 7 (A $\sqrt{ }$ under method name indicates that the risk assessment technique supports the feature). As seen from the results, the proposed method stands out to be fulfilling all the features.

\begin{tabular}{|l|l|l|l|}
\hline $\begin{array}{l}\text { Risk Assessment } \\
\text { Technique }\end{array}$ & Feature & $\begin{array}{l}\text { Tree } \\
\text { Analysis }\end{array}$ & $\begin{array}{l}\text { Proposed } \\
\text { Method }\end{array}$ \\
\hline Risk Prioritization & $\sqrt{ }$ & $\sqrt{ }$ & $\sqrt{ }$ \\
\hline DFMEA \& PFMEA Linkage & & & $\sqrt{ }$ \\
\hline Coupling with Control Plan & & & $\sqrt{ }$ \\
\hline Involvement of External Risks & & $\sqrt{ }$ & $\sqrt{ }$ \\
\hline Ease of Audit & & & $\sqrt{ }$ \\
\hline Relationship to C\&E & & $\sqrt{ }$ \\
\hline Ease of Use \& Maintenance & $\sqrt{ }$ & & $\sqrt{ }$ \\
\hline Reusability & $\sqrt{ }$ & & $\sqrt{ }$ \\
\hline
\end{tabular}

Figure 7: Evaluation of various Risk Assessment Methods

\section{CONCLUSION}

In this paper, the authors have analyzed some of the existing risk assessment techniques and proposed a new mechanism for risk assessment and mitigation. It aims at providing faster results compared to FMEA \& other tree analysis methods. It is also very simple to execute. The concept uses many standard tools in its production.

The technique provides for a total design risk value which can be compared throughout the project life and also to some extent between projects or concepts.

The technique also helps to bridge the gap during development for manufacturing and design to jointly consider the risks. This is because the same risk assessment document and control plan can be used by both design and manufacturing team.

Also, in FMEA out-sourced entities are not included. But with this technique those items will also be addressed. With an FMEA, cause and effect relationship is not considered. This also means that it is difficult to audit an FMEA to verify that the reasoning is valid or to ensure multiple failures or excess complexity is addressed. But with proposed method, C\&E are also included. Hence, it can also be very easily audited. The proposed method tightly couples to Control Plan unlike FMEA which is loosely connected to a Control Plan.

The proposed approach will be run on other case studies. Once the tool is validated, the tool shall be standardized. The standardized tool will be utilized by professional teams in real time projects. The pilot studies done by professionals and application of the technique to real time projects in industries can provide valuable feedback. The shortfalls of the method or suggestions provided by the team shall be incorporated. The future efforts of the authors would include making this approach be fit for use in critical systems such as Nuclear, Chemical and Aerospace industries which use complex tree analysis methods.

\section{REFERENCES}

[1] Failure mode and effects Analysis, http://en.wikipedia.org/wiki/Failure_mode_and_effects_a nalysis, 2014

[2] Design Review Based on Failure Mode, http://en.wikipedia.org/wiki/Design_Review_Based_on_ Failure_Mode, 2014

[3] Failure mode, effects and criticality analysis, http://en.wikipedia.org/wiki/Failure_Mode,_Effects,_and _Criticality_Analysis, 2014

[4] Hazard and operability study, http://en.wikipedia.org/wiki/Hazard_and_operability_stu dy, 2014

[5] Fault tree analysis, https://en.wikipedia.org/wiki/Fault_tree_analysis, 2014

[6] Event tree, http://en.wikipedia.org/wiki/Event_tree, 2014

[7] Cause-consequence Analysis, http://www.safetys2s.eu/modules.php?name $=$ s2s_wp4\&idpart $=4 \&$ idp $=54$, 2014 